\begin{tabular}{|c|c|c|}
\hline \multirow[b]{2}{*}{ CEI I ENT } & Int.J.Curr.Microbiol.App.Sci (2021) 10(08): 140-148 & \\
\hline & $\begin{array}{l}\text { International Journal of Current Microbiology and Applied Sciences } \\
\text { ISSN: 2319-7706 Volume } \mathbf{1 0} \text { Number } 08 \mathbf{( 2 0 2 1 )} \\
\text { Journal homepage: } \underline{\text { http://www.ijcmas.com }}\end{array}$ & 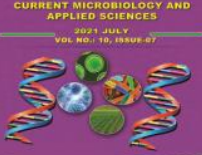 \\
\hline PUBLISHERS & & www. ijcmas.com \\
\hline
\end{tabular}

Review Article

https://doi.org/10.20546/ijcmas.2021.1008.018

\title{
Protection of Maize Varieties under PPV \& FR Act, 2001
}

\author{
Dhirendra Singh ${ }^{1}$, Usha Nara $^{3}$, G. K. Chikkappa ${ }^{2}$ and $\operatorname{Ramesh}_{\text {Kumar }}{ }^{1} *$ \\ ${ }^{1}$ ICAR-Indian Institute of Maize Research, Ludhiana, India \\ ${ }^{2}$ ICAR-Indian Institute of Maize Research, Unit office, New Delhi, India \\ ${ }^{3}$ Department of Plant Breeding, PAU, Ludhiana, India \\ *Corresponding author
}

\section{A B S T R A C T}

\begin{tabular}{|c|}
\hline Keywords \\
\hline $\begin{array}{l}\text { Maize, Registration, } \\
\text { Variety, } \\
\text { PPV\&FRA, } \\
\text { Protection }\end{array}$ \\
\hline Article Info \\
\hline $\begin{array}{l}\text { Accepted: } \\
\text { 12 July } 2021 \\
\text { Available Online: } \\
10 \text { August } 2021\end{array}$ \\
\hline
\end{tabular}

ICAR-Indian Institute of Maize Research (ICAR-IIMR), Ludhiana has been identified as a nodal agency for filing the applications of the public-bred maize varieties for registration under the Protection of Plant Varieties \& Farmers' Rights, Act 2001 (PPV\&FRA, 2001). ICAR-IIMR is selected as a DUS centre for testing of maize varieties with sub-centers at Seed Research and Technology Centre (PJTSAU), Hyderabad; ICAR- Vivekananda Parvatiya Krishi Anusandhan Sansthan, Almora; Punjab Agricultural University, Ludhiana and ICAR Research Complex for NEH Region, Umiam. Till date, 146 applications comprising of 95 hybrids and 51 open pollinated varieties have been filed for protection under this PPV\&FR Act. Out of these, 52 varieties (40 hybrids and 12 open pollinated varieties) fall under new category and 95 varieties (55 hybrids and 39 open pollinated varieties) fall under extant category. The varieties have been registered under new category after completion of two years of DUS testing whereas Variety of Common Knowledge (VCK) category after completion of one year of DUS testing at two locations i.e. Delhi and Hyderabad, respectively. The period of protection is 15 years which starts from dates of registration after completion of DUS testing in case of variety filed under new category. Under extant notified category the duration of protection is counted from date of Gazette notification of the variety.

\section{Introduction}

The agreement on Trade Related aspects of Intellectual Property Rights (TRIPS) especially requires member countries to protect plant varieties. TRIPS provide a choice to the member countries for protecting plant varieties under Patent Act or they can develop a sui generis system or a combination of both.
According to the Sec. 3 (h) of the Patent Act, 1970 , methods of agriculture or horticulture are excluded from patentability. Therefore, in order to protect plant variety in India, the Government of India has established a sui generis system for protection of plant varieties such as "Protection of Plant Varieties and Farmers' Rights Act, 2001'. For the enactment of the Act, the Central Government through its 
notification vide S.O.1589 (E) dated $11^{\text {th }}$ November, 2005 established the "Protection of Plant Varieties and Farmers Rights Authority (PPV\&FRA)" at New Delhi. The PPV \& FR Act has established a legal system for plant varieties protection along with farmers and plant breeder's rights. The plant variety protection has importance in both directions i.e. narrow as well as broad. In narrow direction, plant variety protection increase benefits as incentive to breeders for developing plant varieties. In broad direction plant variety protection has protect farmer's rights, in which farmers can protect their local varieties and wild relatives. Under Section 28 (4) of PPV\&FR Act, 2001, breeder's or licensee or an agent or his successor are become/entitled to produce, sell, market, distribute, import or export of variety registered under the Act. Under this Act, farmer's rights means a group of farmers or the organizations of the farmers are entitled for the registration of the varieties. The section has provided a security for investment in research for developing new varieties. The registration of varieties was initiated on $21^{\text {st }}$ May, 2007 based on the Gazette Notification No. S.O.1884 (E) dated $1^{\text {st }}$ November, 2006. For filing the applications of public- bred maize varieties under new and extant category for registration under PPV\& FR, Act 2001, ICAR-Indian Institute of Maize Research (ICAR-IIMR), New Delhi has been identified as a nodal agency (Jyoti Kaul et al., 2012).

A variety can be filed for protection under new category within one year of seed sale by memorandum of agreement (MoU) to any company/firm as well as within in one year of its gazette notification under Sec 5 of Seed Act, 1966. In another way, a variety can be protected under extant category if it has already completed one year of seed sale or notification. The varieties can also be protected under other categories such as farmer's variety and variety of common knowledge as per their availability. A new and extant hybrid/variety shall be registered under this Act if it confirms the criteria of novelty, distinctiveness, uniformity and stability. Novelty means that such variety has not been sold or disposed of by or with the concerned breeder or his successor. Distinctiveness indicates that it is clearly distinguishable by at least one essential characteristic from any other variety whose existence is a matter of common knowledge. Uniform, if subject to the variation that may be expected from the particular features in its essential characteristics. Stable, if it's essential characteristics remains unchanged after its repeated cycles of cropping. A variety can be registered after successful completion of two years of DUS testing at two locations i.e. Delhi and Hyderabad, respectively. The period of protection is 15 years starts from dates of registration after completion of DUS testing in case of variety filed under new category. Under extant notified category the duration of protection has been counted from date of Gazette notification of the variety.

\section{Application filing procedure}

The documents required for filing the application are given in table 1. Complete application includes the documents such as PV-I, PV-2, Form-I, Technical questionnaire, Dead assignment, GURT affidavit, application fee and registration fee (Table 2). These application forms are also available at PPV\&FR Authority official website (http://www.plantauthority.gov.in).

These forms are required to fill the information for preparation of application for registration. The application form for registration is accepted with specific quantity of seed along with seed quality report as well as registration fee and DUS testing fee (table 2 ). The seed quality report must be issued by a certified laboratory having parameters 
germination (\%), physical purity $(\%)$ and moisture (\%). The specific quantity of seed of the parental lines involved in the development of the hybrid is also required along with the application proforma. The seed quality report enclosed with application form must not be more than one month old. The specific quantity of seed (Jyoti Kaul et al., 2015) and standards (Dass S et al., 2011) are given in table 3 .

Complete application form with all documents signed by the applicant on the bottom of every page, seed, seed quality report and applicable fees as applicable can be submitted at PPV\&FR Authority, New Delhi from Monday to Friday from 10:00 AM to 5:30PM.

\section{Registration Procedure for Protection of Maize variety}

Complete application is received by the PPV \& FR Authority officials, after that officials will issue a PVP number to the applicant.

After issuing PVP number the application is examined by the concern Registrar or Authority officials, if any clarifications are required, queries raised by the Registrar within stipulated time limit (seven days) and to be issued to the applicant for clarifications within stipulated time limit (fifteen days).

After clarifications submission by the applicant on raised queries, if application is accepted, the authority is issue REG number and it is communicate to applicant. After the satisfaction of concern Registrar to dispatch the seed of the variety to concerned DUS centre for two years of DUS testing at two locations in case new category or one year of DUS testing at two locations in case variety of common knowledge (VCK). The registration of extant notified variety is subjected or based on recommendation of Extant Variety Recommendation Committee (EVRC).
The concerned DUS centre conduct DUS testing for new category and variety of common knowledge as per crop specific DUS guideline. During DUS testing of the variety, Chairperson /Registrar/nominee/representative of PPVFR Authority along with a representative/applicant of the variety has to visit the DUS testing sites.

After DUS testing, DUS centre generate and compiled/pooled the data for two years/seasons of two locations with reference varieties and submit the data to PPV\&FR Authority with certification.

Authority will analyze the received data for novelty, distinctiveness, uniformity and stability.

Based on analyzed data report, Registrar to take final decision within stipulated time (fifteen days), the variety may be registered or not.

For pre-grant opposition authority has to publish the case in Plant Variety Journal (PVJ) for invitation of pre-grant opposition. If authority has not received any opposition within stipulated time as per Section 21 (2), then Registry office will be issued registration certificate to the applicant.

\section{DUS testing in maize}

Since 2008, DUS testing has been carried out in maize at two locations i.e ICAR-IIMR, Delhi and SRTC, Hyderabad. DUS testing in maize under new category involves two years of testing at two locations. The category of variety of Common Knowledge (VCK) involves one year of DUS testing whereas farmer's varieties involves grow out test at two locations. The nodal institute and conodal institute has tested large number of varieties from the decade. 
Table.1 Documents required for application filing

\begin{tabular}{|c|c|}
\hline Format & Description \\
\hline PV I & $\begin{array}{l}\text { The form to provide authorization to applicant for filling the } \\
\text { application. }\end{array}$ \\
\hline PV II & The form to provide the information of the variety holders/ developers. \\
\hline Form I & The form to provide registration under new and extant category. \\
\hline Form II & $\begin{array}{l}\text { The form to provide registration under essentially derived variety } \\
\text { category. }\end{array}$ \\
\hline $\begin{array}{c}\text { Technical } \\
\text { questionnaire }\end{array}$ & $\begin{array}{l}\text { The form to provide the scheme and technical information used in the } \\
\text { development of the variety. }\end{array}$ \\
\hline Declaration & The declaration to be signed by the applicant. \\
\hline Deed Assignment & The assignment is signed between Assignee and Assignor \\
\hline GURT Affidavit & $\begin{array}{l}\text { The affidavit tobe signed by the applicant on Rs 100/- stamp paper for } \\
\text { indicating that the variety does not involve Terminator Technology or } \\
\text { the Genetic Use Restriction Technology or any other restriction } \\
\text { technology. }\end{array}$ \\
\hline $\begin{array}{c}\text { Commercial and } \\
\text { Agronomic Attribute }\end{array}$ & $\begin{array}{l}\text { The format to provide the information on commercial and agronomic } \\
\text { attribute of the variety. }\end{array}$ \\
\hline Photo library & $\begin{array}{c}\text { The photo library is required to show the distinct trait/characters of the } \\
\text { variety. }\end{array}$ \\
\hline
\end{tabular}

Source- http://www.plantauthority.gov.in/

Table.2 Detail of fee for registration of maize variety under different category.

\begin{tabular}{|l|c|c|c|c|c|}
\hline \multirow{2}{*}{ S.No. } & Description & \multicolumn{4}{|c|}{ Category } \\
\cline { 3 - 6 } & & New & Extant & VCK & Farmers \\
\hline 1. & Registration fee (Rs.) & $10,000 /-$ & $2,000 /-$ & $10,000 /-$ & Nil \\
\hline 2. & DUS testing fee (Rs.) & $30,000 /-$ & - & $15,000 /-$ & Nil \\
\hline
\end{tabular}

Source- http://www.plantauthority.gov.in/

Table. 3 Detail of required seed quantity and standards.

\begin{tabular}{|c|c|c|c|c|c|c|}
\hline $\begin{array}{c}\text { S. } \\
\text { No. }\end{array}$ & $\begin{array}{c}\text { Registration } \\
\text { category }\end{array}$ & $\begin{array}{c}\text { Seed } \\
\text { quantity } \\
(\mathbf{g m})\end{array}$ & $\begin{array}{c}\text { Germination } \\
\text { capacity } \\
\text { (SCH) } \mathbf{( \% )}\end{array}$ & $\begin{array}{c}\text { Germination } \\
\text { capacity } \\
(\mathbf{D C H}) \mathbf{( \% )}\end{array}$ & $\begin{array}{c}\text { Moisture } \\
\text { content } \\
(\mathbf{\%})\end{array}$ & $\begin{array}{c}\text { Physical } \\
\text { purity } \\
(\boldsymbol{\%})\end{array}$ \\
\hline $\mathbf{1}$ & New Hybrids & 3000 & 80 & 90 & $8-10$ & 98 \\
\hline $\mathbf{2}$ & $\begin{array}{c}\text { Parental lines of } \\
\text { New Hybrids }\end{array}$ & 1500 & 80 & 80 & $8-10$ & 98 \\
\hline $\mathbf{3}$ & Extant hybrids & 600 & 80 & 90 & $8-10$ & 98 \\
\hline $\mathbf{4}$ & $\begin{array}{c}\text { Parental lines of } \\
\text { Extant Hybrids }\end{array}$ & 300 & 80 & 80 & $8-10$ & 98 \\
\hline
\end{tabular}

Source-http://www.plantauthority.gov.in/ and https://iimr.icar.gov.in/ 
Table.4 Details of the varieties filed for registration since 2007.

\begin{tabular}{|c|c|c|c|c|c|c|c|}
\hline \multirow[t]{2}{*}{ Year } & \multicolumn{3}{|c|}{ Hybrid } & \multicolumn{3}{|c|}{ Composite } & \multirow[t]{2}{*}{ Total } \\
\hline & New & Extant & Total Hybrid & New & Extant & Total Composite & \\
\hline 2021 & - & 2 & 2 & - & - & - & 2 \\
\hline 2020 & - & 3 & 3 & - & - & - & 3 \\
\hline 2019 & - & 7 & 7 & - & - & - & 7 \\
\hline 2018 & - & - & - & - & - & - & - \\
\hline 2019 & - & - & - & - & - & - & - \\
\hline 2016 & - & 3 & 3 & - & 3 & 3 & 6 \\
\hline 2015 & 7 & 2 & 9 & - & - & - & 9 \\
\hline 2014 & 6 & - & 6 & 2 & - & 2 & 8 \\
\hline 2013 & 3 & 2 & 5 & - & 1 & 1 & 6 \\
\hline 2012 & 2 & - & 2 & - & - & - & 2 \\
\hline 2010 & 11 & - & 11 & - & - & - & 11 \\
\hline 2009 & 2 & 1 & 3 & 5 & - & 5 & 8 \\
\hline 2008 & 5 & 6 & 11 & 5 & 5 & 10 & 21 \\
\hline 2007 & 4 & 29 & 33 & - & 30 & 30 & 63 \\
\hline Total & 40 & 55 & 95 & 12 & 39 & 51 & 146 \\
\hline
\end{tabular}

Source- Anonymous, Annual Reports (2011-2020)

Table.5 Details of protected public maize varieties under PPV\& FR, Act 2001.

\begin{tabular}{|c|c|c|c|c|c|}
\hline $\begin{array}{l}\text { S. } \\
\text { No. }\end{array}$ & Denomination Registered & $\begin{array}{c}\text { Acknowledgeme } \\
\text { nt Number }\end{array}$ & $\begin{array}{c}\text { Type } \\
\text { of } \\
\text { variety }\end{array}$ & $\begin{array}{l}\text { Certificate } \\
\text { Issue Date }\end{array}$ & $\begin{array}{l}\text { Period of } \\
\text { Protection }\end{array}$ \\
\hline 1 & Amar (D-941) & REG/2007/347 & Extant & 20-Oct-10 & 01-Feb-16 \\
\hline 2 & Aravali Makka-1 (EV-90) & REG/2007/346 & Extant & 02-Jan-12 & 01-Feb-16 \\
\hline 3 & Azad Kamal (R 9803) & REG/2007/415 & Extant & 30-Sep-11 & 01-Feb-20 \\
\hline 4 & BajauraMakka (L 201 Composite) & REG/2008/436 & New & 26-Aug-16 & 25-Aug-31 \\
\hline 5 & BajauraMakka 1 & REG/2009/341 & New & 22-Jan-15 & 21-Jan-30 \\
\hline 6 & Buland (JH-6805) & REG/2007/432 & Extant & 21-Oct-11 & 24-Aug-20 \\
\hline 7 & COBC 1 & REG/2007/418 & Extant & $20-$ Oct-10 & 07-Jun-14 \\
\hline 8 & $\mathrm{COH}-3$ & REG/2007/400 & Extant & 12-Feb-09 & 16-Sep-12 \\
\hline 9 & $\mathrm{COH}(\mathrm{M})-4$ & REG/2007/405 & Extant & $20-$ Oct-10 & 24-Aug-20 \\
\hline 10 & $\mathrm{COH}(\mathrm{M}) 5$ & REG/2007/411 & Extant & 20-Oct-10 & 19-Jul-22 \\
\hline 11 & $\mathrm{CoH}(\mathrm{M}) 7$ (CMH 08-287) & REG/2015/2090 & New & $22-$ Oct-16 & 21-Oct-31 \\
\hline 12 & $\mathrm{CoH}(\mathrm{M}) 8(\mathrm{CMH} 08-292)$ & REG/2015/2091 & New & 22-Oct-16 & 21-Oct-31 \\
\hline 13 & $\mathrm{CoH}(\mathrm{M}) 9(\mathrm{CMH} 08-350)$ & REG/2015/2089 & New & 22-Oct-16 & 21-Oct-31 \\
\hline 14 & $\mathrm{CoH}(\mathrm{M}) 10(\mathrm{CMH} 08-433)$ & REG/2015/2088 & New & 22-Oct-16 & 21-Oct-31 \\
\hline 15 & Composite C-14 & REG/2007/401 & Extant & 21-Dec-09 & 31-Dec-10 \\
\hline 16 & D-994 & REG/2007/343 & Extant & 21-Dec-09 & 30-May-19 \\
\hline 17 & BH-1576(DHM-111) & REG/2010/76 & New & 19-Aug-16 & 18-Aug-31 \\
\hline 18 & BH-1620 (DHM-113) & REG/2010/77 & New & 26-Aug-16 & 25-Aug-31 \\
\hline 19 & DHM 117 & REG/2010/75 & New & 08-Apr-15 & 07-Apr-30 \\
\hline 20 & DHM 119 (BH4062) & REG/2010/327 & New & 19-Aug-16 & 18-Aug-31 \\
\hline 21 & DHM 121 (BH 41009) & REG/2015/1445 & New & 25-Dec-17 & 24-Dec-32 \\
\hline
\end{tabular}




\begin{tabular}{|c|c|c|c|c|c|}
\hline 22 & DMH-2 & REG/2007/416 & Extant & 20-Oct-10 & 03-Sep-17 \\
\hline 23 & Gaurav (D-931) & REG/2007/341 & Extant & 21-Dec-09 & 07-Jun-14 \\
\hline 24 & Gujarat Makai-2 & REG/2007/336 & Extant & 12-Feb-09 & 03-Feb-10 \\
\hline 25 & Gujarat Makai-3 & REG/2007/337 & Extant & 21-Dec-09 & 03-Sep-17 \\
\hline 26 & Gujarat Makai-4 & REG/2007/339 & Extant & 21-Dec-09 & 01-Feb-16 \\
\hline 27 & Gujarat Makai-6 & REG/2007/338 & Extant & 21-Dec-09 & 11-Mar-18 \\
\hline 28 & HHM-1 (HKH-1082) & REG/2007/399 & Extant & 31-Jan-13 & 02-Apr-15 \\
\hline 29 & HHM-2 (HKH-1071) & REG/2007/402 & Extant & 20-Oct-10 & 02-Apr-15 \\
\hline 30 & Him 129 (EHF 1121) & REG/2007/408 & Extant & 20-Oct-10 & 08-Sep-12 \\
\hline 31 & HM-10 (HKH-1200) & REG/2008/438 & New & 30-Apr-13 & 29-Apr-28 \\
\hline 32 & HM-11 (HKH-1237) & REG/2009/364 & New & 04-Dec-14 & 03-Dec-29 \\
\hline 33 & HM-12 (HKH 313) & REG/2012/379 & New & 19-Aug-16 & 18-Aug-31 \\
\hline 34 & HM-13 (HKH-317) & REG/2016/1341 & Extant & 07-Sep-18 & 27-Jan-30 \\
\hline 35 & HM-4 & REG/2007/316 & Extant & $20-$ Oct-10 & 24-Aug-20 \\
\hline 36 & HM-5 & REG/2007/311 & Extant & 12-Feb-09 & 24-Aug-20 \\
\hline 37 & HM-8 (HKH-1188) & REG/2007/317 & New & 16-Мay-13 & 15-May-28 \\
\hline 38 & HM-9 & REG/2007/312 & New & 07-Dec-11 & 06-Dec-26 \\
\hline 39 & HQPM-1 & REG/2007/319 & Extant & $20-$-Oct-10 & 24-Aug-20 \\
\hline 40 & HQPM-4 & REG/2010/263 & New & 26-Aug-16 & 25-Aug-31 \\
\hline 41 & HQPM-5 & REG/2007/310 & New & 07-Dec-11 & 06-Dec-26 \\
\hline 42 & HQPM-7 & REG/2008/437 & New & 05-Feb-13 & 04-Feb-28 \\
\hline 43 & Jawahar Composite Makka-12 (JM-12) & REG/2007/427 & Extant & 02-Jan-12 & 07-Jun-12 \\
\hline 44 & Jawahar Composite Makka-8 (JM-8) & REG/2007/404 & Extant & 02-Jan-12 & 30-Apr-12 \\
\hline 45 & Jawahar Makka-216 & REG/2007/423 & Extant & 02-Jan-12 & 03-Feb-19 \\
\hline 46 & JAWAHAR POP CORN-11 & REG/2008/202 & New & $26-O c t-15$ & $25-$-Oct-30 \\
\hline 47 & KMH-22168 & REG/2013/310 & Extant & 31-Dec-13 & $30-$ Aug-25 \\
\hline 48 & MALVIYA HYBRID MAKKA-2 & REG/2007/398 & New & 27-Dec-11 & 26-Dec-26 \\
\hline 49 & Matunga (DMH-1) & REG/2007/417 & Extant & 02-Jan-12 & 08-Sep-12 \\
\hline 50 & NAC-6002 & REG/2008/41 & Extant & 07-Mar-11 & 03-Sep-17 \\
\hline 51 & NAC 6004 & REG/2008/40 & Extant & 07-Mar-11 & $01-F e b-16$ \\
\hline 52 & NAH-2049 & REG/2009/370 & Extant & 02-Aug-12 & 10-Feb-24 \\
\hline 53 & Narmada Moti (IC- 9001) & REG/2007/340 & Extant & $20-$ Oct-10 & 03-Sep-17 \\
\hline 54 & Pant Shankar Makka-1 & REG/2014/1309 & New & 19-Aug-16 & 18-Aug-31 \\
\hline 55 & Pant Sankul Makka-3 (D 131) & REG/2009/10 & New & 19-Aug-16 & 18-Aug-31 \\
\hline 56 & PAU 352 (JH 3982) & REG/2008/44 & New & $12-F e b-13$ & $11-\mathrm{Feb}-28$ \\
\hline 57 & PMH 1 (JH-10655) & REG/2008/43 & Extant & 21-Dec-09 & $05-F e b-22$ \\
\hline 58 & PMH 2 (JH-3851) & REG/2008/42 & Extant & 30-Sep-11 & 24-Apr-21 \\
\hline 59 & PMH 4 & REG/2010/340 & New & $27-A p r-15$ & 26-Apr-30 \\
\hline 60 & PMH 5(JH 3110) & REG/2010/341 & New & 19-Aug-16 & 18-Aug-31 \\
\hline 61 & PMH 6 (JH 31292) & REG/2014/1310 & New & 26-Aug-16 & 25-Aug-31 \\
\hline 62 & Parkash(JH-3189) & REG/2008/50 & Extant & 30-Sep-11 & 08-Sep-12 \\
\hline 63 & Pratap Hybrid Maize-1 (EH -50802) & REG/2007/315 & Extant & 12-Feb-09 & 03-Feb-19 \\
\hline 64 & Pratap Kanchan-2 (WC-236(Y) & REG/2009/9 & New & 09-Nov-16 & 08-Nov-31 \\
\hline 65 & PratapMakka Chari-6 (EC-3135) & REG/2013/309 & Extant & 31-Dec-13 & 10-Feb-24 \\
\hline 66 & Pratap Makka-3 (EC-3108) & REG/2007/318 & Extant & 07-Mar-11 & 01-Feb-20 \\
\hline 67 & Pratap Makka-4 (EC-1108) & REG/2007/314 & Extant & 07-Mar-11 & 24-Apr-21 \\
\hline 68 & Pratap Makka-5 (EC-3116) & REG/2007/313 & Extant & 02-Aug-12 & 24-Apr-21 \\
\hline
\end{tabular}




\begin{tabular}{|c|c|c|c|c|c|}
\hline 69 & PRATAP QPM HYBRID-1(EHQ-16) & REG/2013/774 & New & 19-Aug-16 & 18-Aug-31 \\
\hline 70 & Priya Sweetcorn & REG/2007/422 & Extant & 20-Oct-10 & 03-Sep-17 \\
\hline 71 & Pusa Composite-3 (Composite-85134) & REG/2007/384 & Extant & 21-Dec-09 & 04-Nov-20 \\
\hline 72 & Pusa Composite-4 (Composite 8551) & REG/2007/387 & Extant & 21-Dec-09 & 04-Nov-20 \\
\hline 73 & Pusa Early Hybrid Makka-1 (EH-202892) & REG/2007/388 & Extant & 12-Feb-09 & 16-Sep-12 \\
\hline 74 & Pusa Early Hybrid Makka-2 (EH 203492) & REG/2007/409 & Extant & $20-$-Oct-10 & 16-Sep-12 \\
\hline 75 & Pusa Early Hybrid Makka-3 (AH-58) & REG/2007/385 & Extant & 21-Dec-09 & 01-Feb-16 \\
\hline 76 & Pusa Extra Early Hybrid Makka-5 (AH-421) & REG/2007/386 & Extant & 21-Dec-09 & 03-Feb-19 \\
\hline 77 & Rajendra Hybrid Makka-3 & REG/2010/304 & New & 23 April 19 & 22-Apr-34 \\
\hline 78 & Shaktiman-1 & REG/2007/428 & Extant & $20-O c t-10$ & 14-Nov-16 \\
\hline 79 & Sakthiman-2 & REG/2007/420 & Extant & $20-O c t-10$ & 03-Feb-19 \\
\hline 80 & Shaktiman-3 & REG/2008/47 & Extant & $20-$ Oct-10 & 19-Sep-21 \\
\hline 81 & Shaktiman-4 & REG/2008/48 & Extant & $20-$ Oct-10 & 19-Sep-21 \\
\hline 82 & Shalimar KG Maize-1 & REG/2007/424 & Extant & $20-O c t-10$ & 24-Aug-20 \\
\hline 83 & Shalimar KG Maize-2 & REG/2008/49 & Extant & 21-Dec-09 & 24-Aug-20 \\
\hline 84 & Co 6 & REG/2013/302 & New & 26-Aug-16 & 25-Aug-31 \\
\hline 85 & Vivek Hybrid-4 (FH-3049) & REG/2007/406 & Extant & $20-O c t-10$ & 07-Jun-14 \\
\hline 86 & Vivek Hybrid-5 & REG/2007/412 & Extant & $20-O c t-10$ & 01-Feb-16 \\
\hline 87 & VIVEK MAIZE HYBRID 39 & REG/2010/217 & New & 01-Feb-16 & 31-Jan-31 \\
\hline 88 & VIVEK MAIZE HYBRID 43 & REG/2010/216 & New & 01-Feb-16 & 31-Jan-31 \\
\hline 89 & Vivek Maize Hybrid 45 (FH 3483) & REG/2013/1286 & New & 19-Aug-16 & 18-Aug-31 \\
\hline 90 & Vivek Maize Hybrid 47 (FH 3513) & REG/2015/1444 & New & 25-Dec-17 & 24-Dec-32 \\
\hline 91 & Vivek Maize Hybrid-15 (FH-3176) & REG/2007/407 & Extant & 12-Feb-09 & 01-Feb-20 \\
\hline 92 & Vivek Maize Hybrid -17 (FH-3186) & REG/2007/413 & Extant & $20-$-Oct-10 & 01-Feb-20 \\
\hline 93 & Vivek Maize Hybrid-21 (FH-3211) & REG/2007/309 & Extant & 07-Mar-11 & 05-Feb-22 \\
\hline 94 & Vivek Maize Hybrid-23 (FH-3529) & REG/2007/410 & Extant & 30-Sep-11 & 05-Feb-22 \\
\hline 95 & Vivek Maize Hybrid-25 (FH 3248) & REG/2007/345 & Extant & $20-O c t-10$ & $04-O c t-22$ \\
\hline 96 & Vivek Maize Hybrid-27 (FH 3288) & REG/2007/344 & Extant & $20-$-Oct-10 & $04-O c t-22$ \\
\hline 97 & Vivek Maize Hybrid 33 & REG/2007/439 & New & 06-Feb-13 & 05-Feb-28 \\
\hline 98 & Vivek Maize Hybrid-9 (FH-3077) & REG/2007/403 & Extant & $20-$ - ct-10 & 01-Feb-16 \\
\hline 99 & VIVEK QPM 9 & REG/2008/198 & New & 17-Jan-13 & 16-Jan-28 \\
\hline 100 & VivekSankulMakka 31 & REG/2008/199 & New & 30-Mar-15 & 29-Mar-30 \\
\hline 101 & VivekSankulMakka 35 (VL 113) & REG/2009/342 & New & 04-Dec-14 & 03-Dec-29 \\
\hline 102 & VIVEK SANKUL MAKKA 37 & REG/2009/343 & New & 07-Apr-15 & 06-Apr-30 \\
\hline 103 & VivekSankul Makka-11 & REG/2007/414 & $\begin{array}{l}\text { Extant } \\
\text { (VCK) }\end{array}$ & 31-Jan-13 & 30-Jan-28 \\
\hline 104 & VLBaby Corn-1 (VL-78) & REG/2007/419 & Extant & $20-O c t-10$ & 01-Feb-20 \\
\hline 105 & Win Orange Sweet Corn & REG/2007/421 & Extant & $20-$ Oct-10 & 01-Feb-20 \\
\hline 106 & Birsa Makai-1 & REG/2007/332 & Extant & 20-Jul-09 & 31-Dec-10 \\
\hline 107 & BirsaVikas Makka-2 & REG/2007/333 & Extant & 20-Jul-09 & 24-Aug-20 \\
\hline 108 & DMRH 1301 & REG/2019/185 & Extant & 12-Mar-21 & 25-Dec-33 \\
\hline 109 & DMRH 1305 & REG/2019/183 & Extant & 16-Mar-21 & 25-Dec-33 \\
\hline 110 & DMRH 1308 & REG/2019/184 & Extant & 02-Mar-21 & 23-Jan-33 \\
\hline 111 & DMRHP 1402 & REG/2019/186 & Extant & 16-Mar-21 & 23-Jan-33 \\
\hline 112 & IMHB 1532 & REG/2019/187 & Extant & 12-Mar-21 & 23-Jan-33 \\
\hline 113 & IMHB 1539 & REG/2019/188 & Extant & 12-Mar-21 & 25-Dec-33 \\
\hline
\end{tabular}

Source: www.plantauthority.gov.in 
Table.6 Detail of fee for maintenance of maize varieties during protection period.

\begin{tabular}{|c|c|c|c|c|c|}
\hline \multirow[t]{2}{*}{ S.No. } & \multirow[t]{2}{*}{ Description } & \multicolumn{4}{|c|}{ Category } \\
\hline & & New & Extant & VCK & Farmers \\
\hline 1. & Annual fee & $\begin{array}{l}\text { Rs. } 2000 /- \text { plus } 0.2 \text { per cent } \\
\text { of the sales value of the } \\
\text { seeds of the registered } \\
\text { variety during the previous } \\
\text { year plus } 1 \text { percent of } \\
\text { royalty, if any, received } \\
\text { during the previous year } \\
\text { from the sale proceed of } \\
\text { seeds of a registered variety }\end{array}$ & $2,000 /-$ & $\begin{array}{l}\text { Rs. } 2000 / \text { - plus } 0.1 \text { per cent of } \\
\text { the sales value of the seeds of } \\
\text { the registered variety during } \\
\text { the previous year plus } 0.5 \\
\text { percent of royalty, if any, } \\
\text { received during the previous } \\
\text { year from the sale proceed of } \\
\text { seeds of a registered variety. }\end{array}$ & $10 /-$ \\
\hline 2. & $\begin{array}{l}\text { Renewal } \\
\text { fee }\end{array}$ & Rs $20000 /-$ (one lump sum) & $\begin{array}{c}\text { Rs } \\
\text { 20000/- } \\
\text { (one } \\
\text { lump } \\
\text { sum) }\end{array}$ & Rs $20000 /-$ (one lump sum) & \\
\hline
\end{tabular}

Source: www.plantauthority.gov.in

Fig.1 A comparative highlights of filing versus protected/registered/certificate issued maize variety.

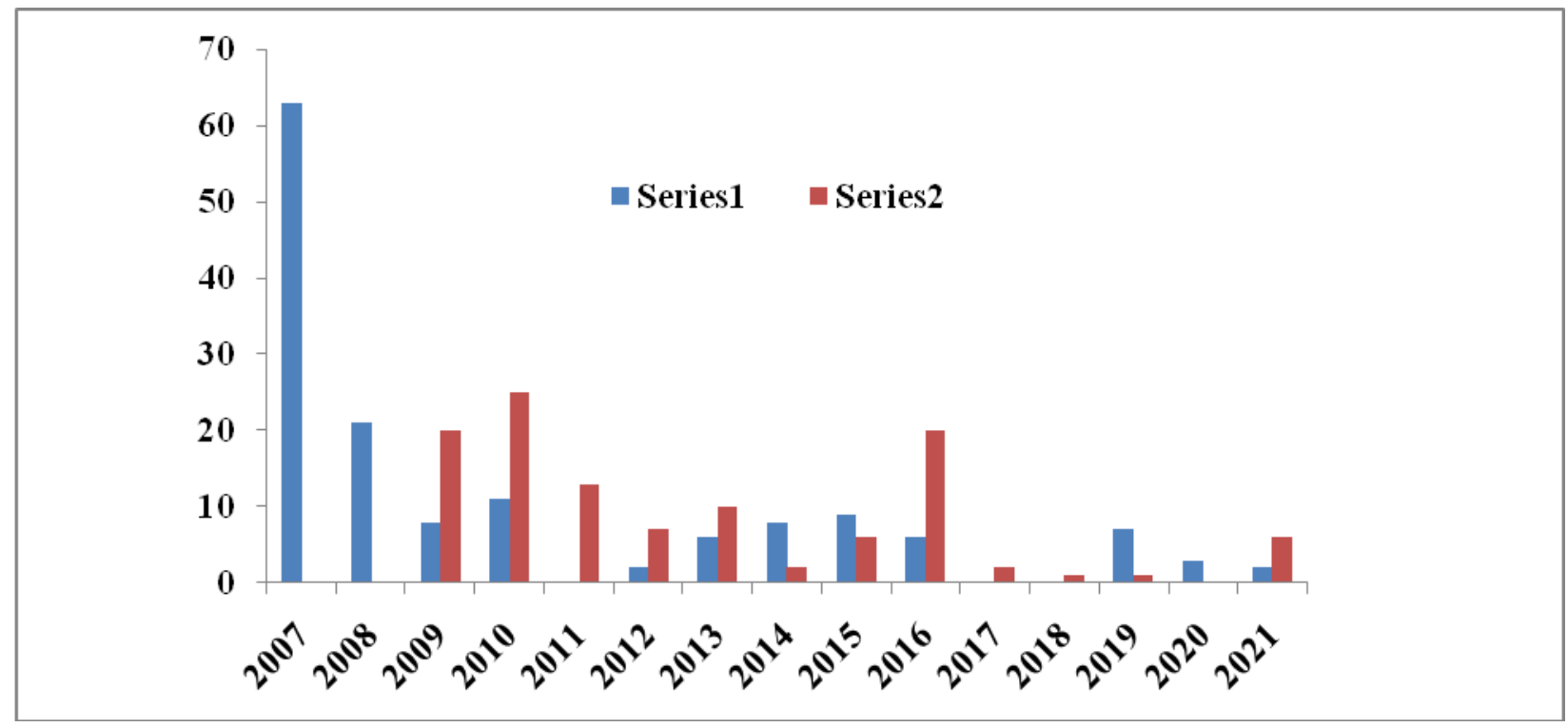

\section{Registration filing details}

In maize, since 2007 registration filing has been carried out at ICAR-IIMR, Ludhiana (formerly known as Directorate of Maize Research, New Delhi).Registration filing in maize involves new and extant category for
Single Cross Hybrid, Multi-parent Hybrid and Composite/Open Pollinated Varieties. The new category is subjected to testing for two years at two locations and Variety of Common Knowledge (VCK) involves one year testing at two locations of whereas, in farmer's varieties one year of grow out test has been 
conducted at two locations. Till date, 146 applications of public maize hybrids and Open Pollinated Varieties has been filed for protection under PPV\& FR, Act 2001.It includes 95 hybrids; 40 under new category and 55 under extent category, 51 composite; 12 under new category and 39 under extent category. The details of yearly filing of applications for registration under various categories are compiled in Table 4.

\section{Registration status of public-bred varieties}

Till date, 146 applications of public maize variety has been filed for protection under PPV\& FR, Act 2001.It involves new, VCK and extant category for Single Cross Hybrid, Multi-parent Hybrid and Composite/Open Pollinated Varieties. This result 113 maize variety are protected till date which includes 40 varieties under new category and 73 varieties under extant category table 5 . The period of protection is 15 years starts from dates of registration after completion of DUS testing in case of variety filed under new category. Under extant notified category the duration of protection has also been counted from date of Gazette notification of the variety. ICAR-Indian Institute of Maize Research has major role in whole process from filing to the applications for registration of all maize AICRP centers. Institute has also maintained the registered maize varieties during protection period. For this, the institute has submitted annual and renewal fees as table 6 for the protected maize varieties from time to time. This promotes scientists for the development of new varieties and its commercialization in Public-Private Partnership (PPP) mode with safe guard. The comparison of registration and filing is shown in diagram 1.

\section{References}

Anonymous, Annual Reports (2011-2020). ICAR-Indian Institute of Maize Research, Ludhiana

ICAR-Indian Institute of Maize Research, Ludhiana official website: https://iimr.icar.gov.in/

Protection of Plant Varieties and farmers Right Authority, New Delhi official website:

http://www.plantauthority.gov.in/

Dass S, Rakshit S, Singh S B, Singh R and Singh I (2011). Guidelines for the Conduct of Test for Distinctiveness, Uniformity and Stability On (Zea mays L.), DMR Technical Bulletin, 2006, pp. 26.

Jyoti Kaul, Ramesh Kumar, Usha Nara, Om Prakash, P. K. Singh (2015). Intellectual property rights for maize varieties of India: A case study. Progressive Agriculture 15 (2): 295299.

Jyoti Kaul, Usha Nara, Om Prakash, Ramesh Kumar, R. Sai Kumar and Sain Dass (2012). Hybrids and composites of maize registered under PPV\& FR Act, 2001. Maize Journal.

\section{How to cite this article:}

Dhirendra Singh, Usha Nara, G. K. Chikkappa and Ramesh Kumar. 2021. Protection of Maize Varieties under PPV \& FR Act, 2001. Int.J.Curr.Microbiol.App.Sci. 10(08): 140-148. doi: https://doi.org/10.20546/ijcmas.2021.1008.018 